\title{
Participatory Design of Therapeutic Video Games for Young People with Neurological Vision Impairment
}

\author{
Jonathan Waddington \\ WESC Foundation \\ Exeter, Devon, EX2 6HA \\ jwaddington@wescfoundation.ac.uk
}

\author{
Conor Linehan, Kathrin Gerling, \\ Kieran Hicks, Timothy L. Hodgson \\ University of Lincoln \\ Lincoln. LN67TS, UK \\ clinehan@lincoln.ac.uk
}

\begin{abstract}
Neurological Vision Impairment (NVI) detrimentally impacts upon quality of life, as daily activities such as reading and crossing the road often become significantly impaired. Therapy strategies for NVI based on visual scanning of on-screen stimuli have recently been demonstrated as effective at improving functional vision. However, these strategies are repetitive, monotonous and unsuitable for use with children and young adults. This project explores the design of a game-based therapy programme that aims to support participant engagement and adherence. We first outline requirements for this software, before reporting on the iterative design process undertaken in collaboration with young people, therapists and teachers at a centre for vision impairment. Our work provides insights into the participatory design of games in collaboration with young people with special needs, and reflects upon the tension of balancing game challenge, therapy goals, and accessibility. Furthermore, it highlights the potential of games to empower special populations by providing a medium through which to communicate the subjective experience of specific impairments.
\end{abstract}

\section{Author Keywords \\ Games; Vision; Rehabilitation; Therapy; Young People.}

\section{ACM Classification Keywords}

K.4.2 [Computers and Society]: Social Issues - Assistive technologies for people with disabilities

\section{INTRODUCTION}

Neurological vision impairment, or vision impairment caused by injury to areas of the brain that are responsible for visual processing, often has a significant impact on functional vision, reducing an individual's independent living skills and negatively influencing quality of life [14,

\footnotetext{
Permission to make digital or hard copies of all or part of this work for personal or classroom use is granted without fee provided that copies are not made or distributed for profit or commercial advantage and that copies bear this notice and the full citation on the first page. Copyrights for components of this work owned by others than ACM must be honored. Abstracting with credit is permitted. To copy otherwise, or republish, to post on servers or to redistribute to lists, requires prior specific permission and/or a fee. Request permissions fromPermissions@acm.org.

34]. Fortunately, effective rehabilitation strategies are available that can help improve functional vision, and lead to improvements in independent living skills [19, 25, 30]. However, successful outcomes typically require months of adherence to daily routines that involve tedious repetitive tasks (e.g., repeatedly scanning images in a systematic manner to find a single geometric shape that differs from other similar distracting shapes) [25]. Adult stroke patients have previously demonstrated adherence to these types of interventions but this has not yet been demonstrated with children [34]. Indeed, these interventions are comparable to clinical assessment tools that have been criticized for being unsuitable and unreliable for use with young people [2]. Conversely, video games have been successfully used to improve excitement and commitment to therapy for young people with a range of conditions [12]. This paper reports on work that explores the use of game design as a means of engaging young people with vision therapy.

In our work, we address the issue of designing vision-based games for people with vision impairment through a participatory design approach. Throughout the development process of our game, Eyelander, we involve young people with neurological vision impairment, and we solicit feedback from therapists. Our work makes three main contributions: (1) We present a novel game-based approach for functional vision rehabilitation, (2) we provide insights into the process of involving young people with vision impairment in the game design, and explore how their needs and abilities need to be considered throughout the design process to create appealing games, (3) we discuss challenges and opportunities to help inform the work of researchers and designers creating games for therapy and rehabilitation through a participatory design process.

Our work is a first inquiry into the design space of visual games for young people with vision impairment, with the goal of providing engaging tools for functional vision rehabilitation. In this context, providing a positive player experience is an important step toward prolonged engagement and improved therapy outcomes, potentially improving independent living skills and quality of life.

\section{BACKGROUND}

This section gives an overview of neurological vision impairment as well as common rehabilitation approaches. Furthermore, we discuss design efforts addressing people 
with vision impairment, and give an overview of games for therapy and rehabilitation.

\section{Rehabilitation for Vision Impairment}

\section{Neurological Vision Impairment}

Neurological vision impairment (NVI) refers to a loss of visual function due to injury to areas of the brain concerned with visual processing. Typical causes of NVI include stroke and perinatal (i.e., immediately before or after birth) asphyxiation. The range of visual processes that can be affected by NVI is wide and they vary depending on the age of the patient, the aetiology of the injury and the neuroanatomy affected. Acquired NVI often leads to fear and isolation; it has a critical impact on skills of independent living such as reading and driving, and significantly affects wellbeing and quality of life. Young people with NVI caused by a perinatal injury will often have a more complex loss of visual perception, such as an impaired ability to see an object when it is placed on a complicated background or when it is not moving [20]. These young people may include those with additional complex needs such as cerebral palsy or learning impairments, but may also include those with no other cognitive or physical impairments [8].

\section{Common rehabilitation approaches}

Until recently, NVI was considered permanent. However, over the last two decades three broad strategies for rehabilitation have been proposed with varying degrees of effectiveness: substitution, restitution and compensation. Substitution involves using a physical device to substitute for a lost area of the visual field. Prismatic lenses can be worn on spectacles to direct the image in the blind visual field towards the healthy visual field [26]. Restitution aims to either restore function to the primary visual cortex or improve visual detection in nearby extra-striate visual pathways. This is typically undertaken with visual stimulation using extremely bright, high contrast stimuli [17]. Compensation aims to change behavior to compensate for lost visual function. This is typically achieved by developing efficient eye movement strategies during training with visual arrays and active scanning tasks [22, 25] (see figure 1). The current project focuses on building tools that teach and encourage compensatory strategies.

\section{Designing Games for Vision Therapy}

In order to understand the requirements of games for vision therapy we must consider existing research on designing video games for people with vision impairment, as well as research on using games for therapy and rehabilitation.

\section{Designing video games for people with vision impairment}

A growing body of research is beginning to focus on the development of video games that are accessible to players with vision impairment. Morelli et al. [23, 24] present a series of accessible versions of commercially available games (e.g., Nintendo Wii Sports Bowling and Tennis). In their games, the authors replace visual cues with auditory and tactile feedback. Following a similar approach, Rector et al. [27] explore the creation of an eyes-free yoga exergame that provides balance training for people with vision impairment and builds on audio cues to guide player movement.

In contrast, Eriksson et al. [9] present mini games that were designed for children who are blind and for players with less severe vision impairment, with the goal of encouraging children to practice the recognition of simple visual objects; results of user studies show that visual cues are considered helpful by players with a wide range of visual abilities. Likewise, Ching et al. [5] explore the inclusion of graphical content for players with vision impairment in their project Beachcomber. The game builds on touch- and audio-based interaction and includes high-contrast graphics; initial playtests suggest that players with vision impairment appreciate the graphical dimension of the game. Thus, existing work on video games for people with vision impairment show that it is possible to design video games that are accessible for players with vision impairment.

\section{Games for therapy and rehabilitation}

Recent years have seen much interest in the use of games technology to improve the engaging qualities of therapy programmes (see [16]). For example, Kato et al. [18] have demonstrated that games can be an effective means of increasing compliance [18]. Similarly, Achtman et al., found that games can help patients follow through with otherwise tedious therapy routines [1]. Games have been applied to teach sign language to deaf children [15], to support children with autism [11] and to provide cognitive training for children with Fetal Alcohol Syndrome [3].

There are a number of design lessons to take from these studies. Firstly, the justification for using games in therapy is typically based on the ability of games to improve participant motivation to engage with often-repetitive therapy tasks. In order to generate this type of motivation, games must present challenges to participants [29]. Goals are set through the game narrative and players aim to reach that goal by engaging in therapy appropriate behaviour. Secondly, when training a skill via game play, it is crucial that the target skill must form the basis of the core game mechanic $[10,13]$. For example, if the therapy is designed to improve dynamic balance (i.e.,[10]), the principal way in which players interact with, achieve success in, and progress through, the game should be through measures of their ability to balance. Thirdly, a user-centered design approach that also takes into consideration the needs of therapists is essential [6].

\section{Applying games in vision training and therapy}

The use of games, a primarily visual medium, for the purposes of vision therapy presents a number of interesting opportunities. For example, there is some evidence that video games can encourage users of all abilities to follow 
through with tedious visual tasks [9]. Additionally, there is some evidence that video games have the potential to affect visual skills of participants of all abilities [1, 4, 11, 14]. For example, Li et al. [21] showed that playing video games has positive effects on how people with impaired spatial vision perform in functional vision tests. However, Achtman et al., [1] in a review article, concluded that there is little evidence that the use of commercially available games in therapy leads to improvements in vision that transfer to daily life. To facilitate this transfer, it seems crucial to develop games that strictly follow therapy requirements, rather than applying existing products.

While custom-designed video games appear a promising means of encouraging young people with NVI to participate in rehabilitation, there is no record in the literature of previous projects that have attempted to meet this goal. In this context, one of the core challenges of the work presented in this paper is translating therapy requirements into effective, yet engaging game mechanics that have the potential of eliciting the same type of visual (i.e., eye movement) behaviour as traditional therapy, while providing an enjoyable game experience.

\section{Design Requirements}

\section{Interface and visual design}

The use of a visual medium for the purposes of vision therapy presents notable challenges. Research on design for people with vision impairment typically focuses on the substitution of visual interface elements [23, 24] with stimuli that address other senses (e.g., hearing, touch). This approach is not appropriate in the context of vision therapy. Functional vision rehabilitation builds on the presentation of visual cues to encourage people with vision impairment to develop compensatory strategies such as eye and head movements to improve the effectiveness of visual scanning. To achieve this goal, players must complete search tasks using their visual skills, and displayed images should not be completely replaced with haptic or audio interface elements. The interface should be primarily visual, thus a standard PC, monitor and mouse will be used.

\section{Requirements of vision therapy}

Tools previously used for training compensatory visual scanning techniques are derived from stimulus arrays used in cognitive neuroscience experiments [25] (see figure 1). Simple geometric shapes are presented in white on a plain black background. Training requires the participant to passively view these stimulus arrays, searching with eye movements for a target stimulus in an array of distractors. The target typically differs from distractors on one physical dimension, such as size, shape or orientation. Each scanning task remains on-screen for 3 seconds. There is no mechanism for the participant to indicate whether or not they found the target stimulus. Each therapy session requires participants to carry out these scanning tasks for approximately 20 minutes and sessions must run 4-5 times per week for a number of months.

While adult stroke victims have demonstrated adherence to this intervention [25], due to its repetitive and passive nature it does not seem appropriate for use with young people. There is significant opportunity to use games design elements to improve the engaging qualities of these tasks. Indeed, simply improving the visual design and introducing interactivity would presumably help. A further complication is that there is little evidence to explain which specific features of search tasks are essential for successful therapeutic outcomes, and which are not. Thus, for pragmatic reasons, it seems necessary to replicate as much as possible in our game the functional characteristics of those original search tasks.

\section{Therapy-appropriate game mechanics}

When training a skill via game play, it is crucial that the target skill must form the basis of the core game mechanic $[10,13]$. In our game, the core mechanic should involve the repetitive visual searching of an array of distractor stimuli in order to find a target, since this is the behaviour that is targeted by existing vision therapy. Notably, one of the core tasks in our participatory design process is exploring the tension between the design goals of challenge and accessibility. Vision therapy is inherently challenging for people with vision impairment. Games must also present game challenges for players in order to achieve their motivational qualities [30]. Both of these types of challenge have the potential to frustrate participants and players, and could lead to disengagement with the design process, or with the resultant game.
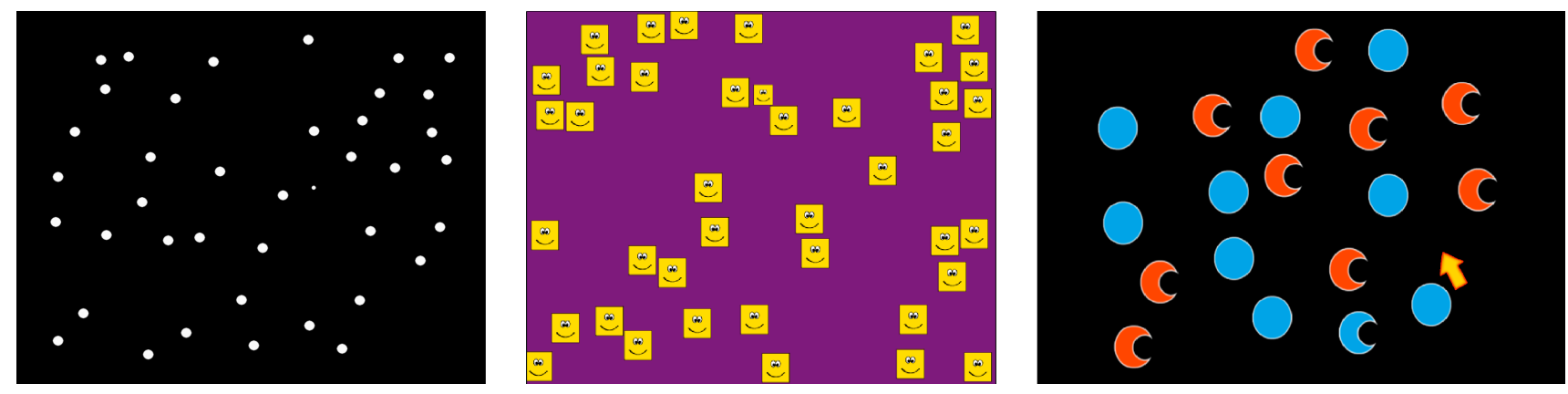

Figure 1. The original search task (left), an early version of the game (middle), and final version included in Eyelander (right). 


\section{End user adaptability}

Due to the wide range of abilities observed with users of therapeutic games, it is essential to allow adaptability of game features based on player ability [10]. For example, through the process of developing game-based rehabilitation technology for adult stroke patients, Flores et al., [10] found that it was important to allow the user, carer, or therapist (as appropriate) the ability to adapt complexity of both the visual content and the cognitive challenges presented by the game. In addition, the game should allow the therapist, carer and player to track performance over time, in order to allow for intuitive judgments over whether progress is being achieved [7].

\section{A PARTICIPATORY DESIGN APPROACH TOWARD DESIGNING GAMES AS VISION (RE)HABILITATION THERAPY FOR YOUNG PEOPLE}

Due to the large number of requirements outlined above, it was not appropriate to begin the participatory process entirely from a blank slate. Rather, an initial prototype was developed, following the above guidelines, which formed the focus of sessions run with participants.

\section{Initial Game Design}

At the beginning of the design process, we focused on the development of a core mechanic that would allow us to integrate therapy requirements into Eyelander. This section focuses on the core mechanic. The theme and narrative of the game evolved throughout the design process and is described in the next section.

The core game mechanic, taken from previous research, required players to visually search arrays of coloured shapes in order to find specified target stimuli, and to click on those targets. Requiring players to make a click introduced interactivity, which is absent from the traditional search tasks, and supported the delivery of performance feedback. Correct clicks triggered both audio effects, and occasionally visual effects. The search tasks were framed by simple animations that introduced the concept of progression to the game, as a means for introducing challenge, goals and driving longer-term engagement. The player was presented with a goal for the play session (i.e. complete ten numbered levels) and medium term goals (i.e., a character climbing a ladder), which helped track progress.

\section{Participants}

We recruited three groups of participants for the iterative design sessions. Our first group included young participants with a diagnosis or presentation of NVI. We recruited four learners ( 3 female, 1 male), aged 15-18 from a specialist education center for learners with vision impairment. Two participants had a complex neurological presentation with suspected NVI and associated developmental delay, learning, speech and language difficulties. One participant had no light perception in the left eye and a right sided visual field loss caused by optic glioma. Our fourth participant had a confirmed diagnosis of cerebral palsy, left hemiplegia and suspected NVI. Two participants were legally blind and two had low vision.

A researcher met with this group of participants during fortnightly meetings over a three month period held in a familiar room at the centre. Each meeting lasted approximately half an hour, during which time participants were asked to play test the game and provide feedback on the game's visuals, controls and mechanics. While one of these participants gave consent to audio recording, two did not, and the fourth was non-verbal. Therefore, data from this group of participants was predominantly collected by observing behavior and note taking.

We recruited two further groups of participants for separate one-off design sessions. Our second group of participants included the therapy team that worked at the same specialist education center as our first group of participants, consisting of occupational, physical, and speech and language therapists ( 8 total) who had daily experience working with learners with NVI. We invited this group so that we could understand the concerns of gatekeepers, and also to come to solutions that our first group of participants may not have been able to provide alone. Our third group of participants consisted of three young people with acquired brain injury and suspected hemianopia who were part of a local community group. Each person in this group of participants had relatively minor cognitive and physical impairment.We felt that conversations with more cognitively abled participants would help us to corroborate and expand upon what we learned during observations with our first group of participants.

Data was collected from the second and third group of participants during two separate one-off design sessions that lasted approximately two hours. A researcher met with the second group of participants on the fourth week of the iterative design process, and with the third group of participants on the fifth week. A brief for the project was explained to each group, and participants were invited to play and observe the current iteration of the game at that time. After a roundtable discussion, each group collated a list of collectively agreed considerations and suggested amendments to the game. These findings were implemented gradually through the iterative design process.

\section{Iterative Design Sessions}

Observations are organized according to the requirements outlined above. The changes to game features implemented between the major iterations of the game are outlined in figure 2. These changes all emerged from findings of participatory design sessions.

\section{Visual design of search tasks}

Our first attempt at making the game more visually engaging was to add colours and smiley faces to the shapes displayed in the visual search task (see figure 1). While the range of different colours were appreciated, the additional 


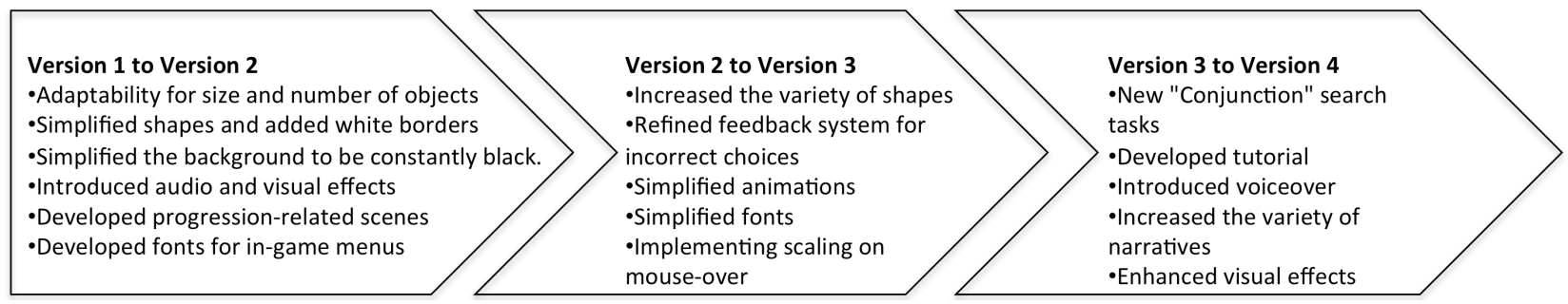

Figure 2. Flow chart describing the features designed and implemented across the major iterations through participatory design process.

detail of the smiley faces was either too difficult to see or queried negatively by participants ("What is that?"). We also found that the size of the shapes used in the original visual search task were too small for some of our participants to see.

In the next iteration we increased the average size of shapes and added an option to change the average size to "small", "medium" or "large". To increase contrast we kept a black background and added a white border around the edge of each shape. Engagement during this iteration dramatically improved. Negative feedback was rare from our first group of participants. However, they regularly requested more variety in shapes and colours. The original visual search task used lines, dots and squares. We increased the variety of geometric shapes through iterations, with the final version including rectangles, circles, squares, triangles, crescent moons and five-pointed stars.

\section{Visual design of narrative scenes}

The scenes that introduce narrative and illustrate player progress are more visually complex than the search tasks. We observed that these screens could pose a problem for some of our players. Specifically, persons with NVI can have difficulty with visually processing and identifying objects that they haven't seen before. To address this we added a number of features. Firstly, we introduced voiceovers for the level selection screen. When a player hovered the mouse cursor over a selectable level, a voiceover described the level in a short quirky phrase (e.g., "Barry the Alligator's Swamp") so that players have some prior information and preparation before they see the level. Secondly, when the player starts a level, only the avatar is visible on a black background. After a very brief period of time $(500 \mathrm{~ms})$ we fade in the obstacle that they have to overcome e.g. lava rises from underfoot, and finally after another period of time $(500 \mathrm{~ms})$ we fade in the background. This gives the player time to locate each of the key objects in the scene: the avatar, the obstacle, and the background rather than having to process the image all at once.

\section{Visual design of menus}

We attempted to introduce different styles of fonts to judge which participants might prefer. The participants in our first group were able to read all of the fonts, but during our meeting with the second group of participants (the therapy team), they recommended that we stick with a sans-serif
(Arial) white font. We updated the font for the next iteration of the game and our first group of participants commented that the text was much clearer so we continued using the simple font.

\section{Diversity in game mechanics}

Our third group of participants, who were cognitively able, found some of the silly aspects of the game engaging (e.g. the stampede of angry cows), but felt that the game mechanics would get boring after time. These participants suggested new mechanics, such as having to remember a number of different shapes in a sequence that the player would have to find. While this seemed like a useful suggestion for the more cognitively able users, we were concerned that this might make the game inaccessible for those with cognitive impairment and may also be too different to the original visual search task and so might change the effect of the therapy. As a compromise we added more complex search tasks, where multiple groups of distractor shapes would be generated on the screen at any one time ("conjunction task"). This interaction demonstrated the difficulty involved in designing games for participants with a wide range of cognitive and physical ability. Games must be accessible for all users, while still challenging for those with more developed abilities.

\section{Engagement}

Three of the four participants in our first group played the game through to completion each session (111 search tasks; 20-30 minutes). Two of these participants were excitable throughout (e.g. observed bouncing on their seat, and clapping their hands when correctly identifying objects), and enthusiastically asked to play again when finished. On separate occasions we allowed these two participants to play through the game more than once, and they continued to play until the researcher ended the session after 45 minutes. One participant dropped out of the study after two sessions. This participant was non-verbal, so we were unable to discern the precise reason for disengagement. However, this person was clearly disinterested during play sessions, only completing 3 or 4 levels each play test before going back to his iPad or web browser.

\section{Conclusions from iterative design sessions}

One of the conclusions derived from the above interactions was that design features to support accessibility should be utilised during all sections of the game other than the search 
tasks. Although the therapy required players to use their visual skills to complete search tasks, it was not necessary for players to use their vision to perform other actions in the game such as selecting gameplay options from a menu. This was something that we missed in our initial requirements generation, due to the design goal of forcing participants to use their visual skills. This interaction also illustrated the usefulness of consulting experts in the therapy team. While participants were able to engage with the original designs (presumably with some difficulty), they did not have the knowledge or expertise to explain to us that there was a preferable solution. Participants can help us understand whether design features are acceptable, but may not be able to help identify solutions that we have not already considered.

A surprising finding was that even experts may not have a complete understanding of the subjective experience of vision impairment. During the session with the therapy team, some members asked how learners who were legally blind had coped with the game. They reacted with stunned silence when informed that the participants had been able to play the game to completion a number of times. Indeed, therapists seemed genuinely concerned that the visual media in the game would be too difficult, complicated or unfamiliar for participants with NVI to see, although others thought that more varied visual rewards could improve engagement. This illustrated the complexity involved in designing visual games for visually impaired people.

\section{Final Game Design}

\section{Game mechanics and narrative}

Core game mechanics remained relatively stable across iterations, due to the therapeutic requirement to strictly adhere to the formal components of the visual search task. However, through iterations, we focused on developing a coherent game narrative with which to frame the search tasks, with the intention of presenting a coherent and challenging goal, and helping participants to monitor their progress towards that goal. The final version is significantly more complex and rich than the original version, which simply had numbered levels and an animation of a character climbing stairs to denote progress.

When a player begins a new game they are presented with a brief narrative that frames the long-term goal for the session. The basic narrative is to help a character escape an island being engulfed in lava after a volcanic eruption. There are four additional scenarios that can occur, which impede the character's escape: stampeding angry cows, an invading UFO, a "naughty" panda, and a massive baby that blocks the character's path. Players are presented with a level selection screen illustrating the pathway through the levels from start to finish (see figure 3). Only the most recently unlocked level can be selected, and the player is given audio and visual cues when the cursor is over this target.

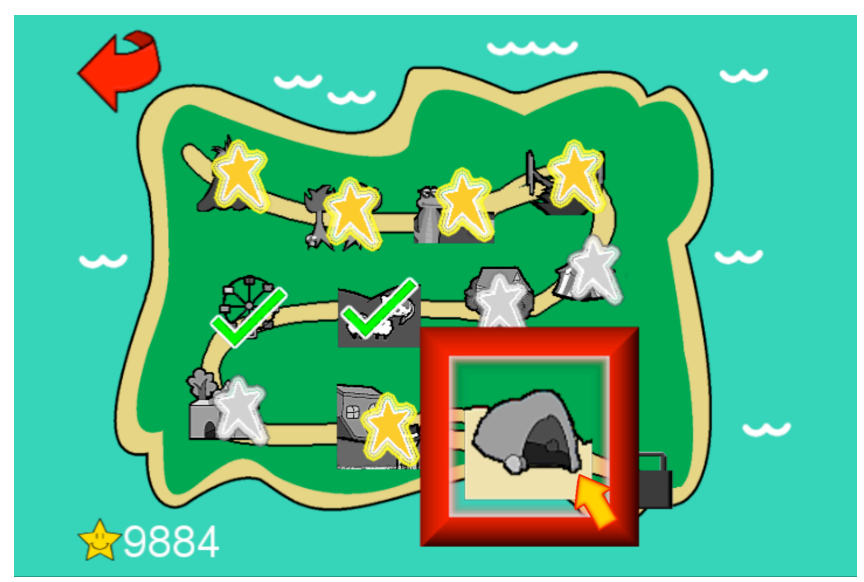

Figure 3. The island screen used to help participants track their progress through game sessions.

Each level begins with a simple animation that sets the goal for that level (i.e. overcome an obstacle such as a river of lava). Each visual search task begins with a description and visual presentation of the target shape that the player is asked to find. Targets are generated with randomly assigned features: shape, size, colour, orientation, and "blinking", and then placed at a random location on the screen. A group of distractor shapes are generated that differ from the target by one randomly chosen feature (again; shape, size, etc.), and are then placed at random locations on the screen such that they don't overlap. If the player selects the target shape on the first attempt they receive positive feedback through audiovisual effects and the game progresses. If the player selects a distractor on the first attempt nothing happens, and if they find the target after that the game progresses but does not give audiovisual feedback.

Once the player has completed the predetermined number of visual search tasks for that level they are rewarded through an animation of the character overcoming the obstacle that impeded progress earlier. After the player has completed a level they return to the level selection screen and can view their progress as visual cues mark where the player's character has already been and how well they performed (e.g. a gold star is displayed for finding all the target shapes first time on a level). Each successive level contains progressively more search tasks, and progressively more difficult search tasks. "Dummy" tasks (where the target shape does not appear) are added randomly with $10 \%$ chance from level 5 onwards, and the player must click the right mouse button to complete these tasks. At the end of the game the player is congratulated and presented with some feedback on how well they did and a high score table to compare with previous attempts.

\section{End user adaptability options}

We have developed three key options that can be changed depending on the varying visual, cognitive and physical abilities of players. These options include the average size of target shapes, the limit on the number of shapes 
displayed, and the time limit before a search task resets. The average size of shapes can be adapted to be "small", "medium", or "large", approximately equivalent to individual shapes covering $2 \%, 7 \%$ and $15 \%$ of the viewable screen area. Increasing the size of shapes was necessary for those participants with low vision to access the game, but decreasing the size of shapes can be useful for participants with extremely restricted visual fields. The option to limit the number of geometric shapes displayed during a search task can be adapted for those with impaired visual attention or an impaired ability to discriminate between multiple targets displayed at the same time (simultanagnosia). The time limit before a search task resets can be increased to give additional time to those players who require longer to process visual information or to physically move the mouse to respond.

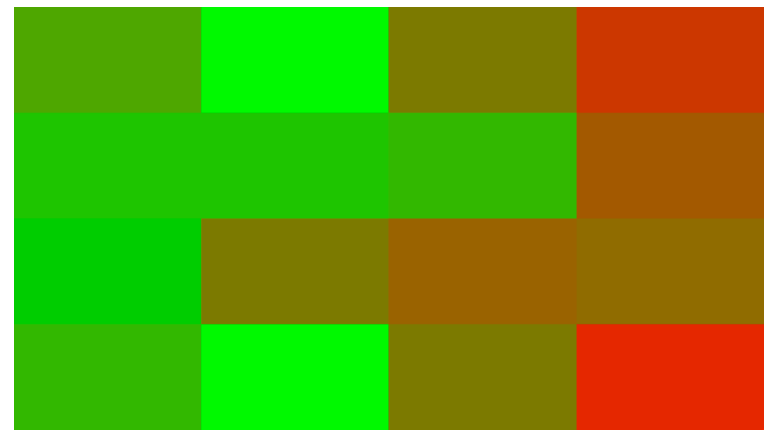

Figure 4. Example output of the "heatmap" style automated assessment tool.

\section{Automated assessment tool}

We have also developed an automated vision assessment within the game, based on dividing the screen into a $4 \times 4$ grid that represents the participant's visual field limited by the size of the game screen. The automated assessment tool records the number of correct responses, incorrect responses, and response times to find targets in the game within each respective area on the $4 \times 4$ grid (not including "dummy" trials). As images are randomly distributed with a 2D uniform distribution on the screen during search tasks, it is possible to gather this data from each area of the grid if the player attempts enough tasks. The "heat map" generated from data recorded in this manner can help healthcare professionals assess the functional visual field of the player and pinpoint any problem areas (see figure 4). For example, if a player consistently makes "incorrect" responses on the left hand side or if they always take much longer to respond on this side, this may indicate a left visual field loss or impaired spatial attention. This assessment was not possible in the previous tools for visual search training, which did not have an interactive element. Visual field assessment of young people with NVI is notoriously difficult to perform. Collecting data over the rehabilitation period in this manner could help inform healthcare professionals about those problem areas and whether the training has been effective at improving the response to targets displayed in those areas.
However, it is still necessary to assess whether functional vision skills learned in-game can translate to other activities of daily life.

\section{Formative Longer Term Study}

Since one of the core goals of the project is to promote longer term engagement with the therapy, we found it valuable to include within the participatory design process, a formative playtest with the prototype that ran over a number of consecutive days with the same participant. This participant was a 20 year old female, with a diagnosis of right sided hemianopia and no perception of light from the left eye, with very minor cognitive and physical impairments. She had a very constricted visual field but moderate visual acuity in her remaining visual field.

This participant played the game for four consecutive days one week, followed by two consecutive days the following week, with the first week closely matching the expectations of the final therapy programme. Observations during these sessions provide a number of interesting insights. Firstly, the participant always turned the music and audio off after the first level, reporting that it was "okay" but could get distracting and annoying. Secondly, the game was played through to completion every time, representing 20-30 minutes of game play. This is an encouraging result in light of the goal of player retention. However, it must be noted that different behaviour may have occurred if the participant was not being observed by the researcher. Thirdly, the participant sometimes adopted a head turn slightly to the right, presumably so that a larger area of her functioning visual field was facing the screen. It is currently unclear whether this is therapy appropriate behaviour, as emphasis in previous rehabilitation studies has focused on investigating eye movements only and not gaze. As the game is designed to promote effective eye movement strategies but eye movements are not measured directly it is possible that players may develop other behaviours to complete the game that are not therapy appropriate.

Interestingly, the heat map recorded on the first day of play gave a good indication of the participant's visual field, which is characterized as a thin band of vision from the midline to $45-60^{\circ}$ left of the midline. The largest number of missed shapes and longest reaction times were on the far right, whereas all the shapes just left of the middle were found first time. This is an early indication that our automated assessment tool may prove useful in giving feedback on functional vision to players, carers and healthcare professionals.

Over the first four days both the participant's response time and the percentage of targets found continued to improve from $3.24 \mathrm{~s}$ and $86 \%$ respectively to $2.30 \mathrm{~s}$ and $99 \%$. There was a dip in accuracy at the start of the second week but this improved on the final day when the participant had an average response time of $2.18 \mathrm{~s}$ and found $95 \%$ of targets first time. These are encouraging results, although a 
controlled trial including measures of functional vision will be required to draw conclusions on the therapeutic effects of the training.

\section{DISCUSSION}

This paper outlines design requirements and presents the participatory design process of Eyelander, a video game to engage young people with NVI in functional vision rehabilitation. In this section, we discuss the implications of our findings for the design of video games for persons with vision impairment, we reflect on the role of challenge in game design for therapy and rehabilitation, and we highlight areas in which our findings can be generalized beyond game development for children and teenagers.

\section{The Design of Interactive Interventions for Vision Rehabilitation}

Visual simplicity and accessibility vs. visual appeal

The most important advantage of involving end-users in the design process of Eyelander was that it allowed us to tailor the graphical design of the game to the abilities and preferences of the target audience. The resulting design is rather simplistic (see figure 1 and figure 3 ), but our results show that this ensures accessibility to a range of players. While players generally expressed a positive view on the visual design of Eyelander, feedback from other stakeholders (e.g., parents and therapists) revealed a challenge for game designers: persons without visual impairment expressed concerns regarding the visual appeal when comparing Eyelander to commercially available games. Communicating the importance of accessibility and the implications for visual design - to these stakeholders therefore seems to be an important step to ensure system adoption, as we expect parents and therapists to function as gatekeepers regarding the introduction of games to support vision therapy.

\section{Games as an opportunity to reveal player abilities}

Much research on games for players with disabilities particularly on games for persons with visual impairment focuses on ways of designing around the disability to provide a positive player experience (e.g., [23, 24]). Due to the therapeutic background of Eyelander, it was not possible to follow this approach; while this raised concerns about the possibility of exposing player vulnerability through visually challenging play (as discussed in the following section), the design sessions also revealed the potential of the game to highlight player abilities in a positive context. In the case of NVI it is often difficult to develop a detailed understanding of the visual abilities of a patient as the effects of impairment are often complex and highly individual. Eyelander helped therapists understand the individual visual capabilities of patients by giving players a platform on which they could demonstrate visual competence. In this context, the fact that therapists expressed surprise regarding the abilities of some players highlights the potential of as a means of empowering players with disabilities by allowing them to share abilities with others through play.

\section{Player Abilities, Therapy Goals, and Role of Challenge in a Participatory Design Process}

A recurring theme during the participatory design process of Eyelander was the importance of designing for challenge in games. In this section, we discuss how in-game challenge and therapy-related challenges need to be consolidated, and we discuss the role of challenge in games in the context of participatory design with audiences with special needs.

\section{In-Game challenge and challenges related to therapy}

There are two distinct types of challenges presented by a therapeutic game to the player. Firstly, the therapeutic task itself will represent a challenge for the player to undertake. Therapy necessitates repetition of skills or behaviours that the player is not proficient or comfortable in performing. Secondly, in order to present engaging and meaningful experiences for players, games must present appropriate levels of game challenge to participants [32]. These ingame challenges are the central means through which games generate the motivation and engagement that is valuable in the context of therapy. Both of these types of challenge can function as a source of frustration for players, and have the potential to undermine the engaging qualities of the game. Designers of therapeutic games must consider and address both types of challenge simultaneously when defining in-game tasks.

Further complicating the design task, game and therapy requirements can sometimes be contradictory. The activities necessary for successful therapy are not always (or often) the most fun feature to be implemented into games. For example, we initially planned to develop a series of interconnected but distinct mini-games. Upon researching the neuroscience literature in more detail, and realizing that the mechanics behind visual therapy are not yet well understood, we realized that we could not justify the design of tasks that were significantly different than the basic visual search tasks that were previously therapeutically successful. In another example, playing the same game every day for a number of months is probably not what participants would choose to do. Nonetheless it is therapeutically necessary. Generally, therapy may involve overstraining patients and pushing them to work at the limits of their abilities, whereas games try to balance challenge and ability to allow optimal experience [29].

\section{Participatory design and challenge}

Additional problems can be introduced when using a participatory design approach to design games that offer adequate levels of challenge.

Challenge vs. game accessibility. As demonstrated in our work, engaging in a collaborative, iterative design process is crucial in understanding the types of challenges experienced by participants while playing a therapeutic 
game. Only through this process can the designer make an informed and compassionate decision on which of the frustrating or uncomfortable tasks that may be presented by the game are unavoidable and necessary, and which may be avoided or supported through accessible design techniques. Through our design sessions we quickly realized that we needed to make all of the game elements surrounding the search tasks as accessible as possible, while maintaining the inherently challenging nature of the search tasks.

Participatory sessions also led us to two further insights that contradicted initial assumptions. Firstly, young people who had been declared legally blind, and who therapists and teachers did not expect to be able to play anything other than a hugely simplified version of the game, actually displayed little problem in playing the game successfully. Thus, we were able to set the baseline challenge of the search tasks far higher than we initially expected. Secondly, we found that young people often did not mention when they found something really difficult, as long as they were in any way able to engage with it. Often it was necessary to consult with the therapy team to understand how to improve our designs as the young people simply refused to complain.

Exposing vulnerability when trying to design challenging experiences. One of the drawbacks to using a participatory design approach is the significant potential, as a by-product of the process of exploring and testing activities that participants find challenging, for the exposure of participant vulnerabilities. During design sessions we must ask questions of our participants regarding whether they can see the on-screen items and whether they can discriminate between different shapes, since this is the goal of the scanning task. Designers in this situation, as in any situation working with vulnerable participants, cannot avoid reminding participants of the limits of their abilities, something that has the potential to cause upset.

\section{LIMITATIONS AND FUTURE WORK}

While the focus of this paper is on the design process of Eyelander, a limitation in terms of assessing the effectiveness and the appeal of the proposed approach is the lack of a long-term user study to investigate player experience, engagement, as well as therapy outcomes. We intend to assess how feasible and effective this computer game could be as a rehabilitation strategy in a formal longterm study. Additionally, our approach strongly focuses on younger players; while our design focus was focused on young people, game-based visual rehabilitation may also be valuable for other persons, e.g., adults. In this context, it would be valuable to explore their preferences and needs in terms of game design.

\section{CONCLUSION}

Recent research suggests that video games can be an effective means of supporting therapy and rehabilitation. However, the success of games in this context depends largely on their potential to engage and motivate users. Designing enjoyable games is a challenging task, made significantly more complex when the manner through which participants perceive and interact with the game is significantly and qualitatively different to that of the designers. The participatory design process of Eyelander allowed us to explore how the involvement of young people with vision impairment can contribute to design of games to support vision (re)habilitation. One of the core challenges identified in this process, and one that must be dealt with in the design of any therapeutic game, lay in judging the balance between presenting the player with (necessary) therapeutic, and (motivating) game related, challenges, while ensuring that accessibility guidelines are considered and players are not unnecessarily frustrated. Furthermore, we found that the process of finding this balance was sometimes problematic, potentially exposing participant vulnerabilities. However, through playing the game in play test sessions, players were also able to demonstrate and communicate their specific visual abilities in ways that often stunned therapists and tutors who worked with them on a daily basis. This experience highlights one of the powerful benefits of games for special populations; they can help people to communicate to others the subjective experience of living with their specific impairment.

\section{ACKNOWLEDGEMENTS}

We acknowledge the time contributed by all participants. This work was supported by The WESC Foundation, the Technology Strategy Board, and the Medical Research Council of the United Kingdom, as part of a Knowledge Transfer Partnership (Ref: KTP008989).

\section{REFERENCES}

1. Achtman, R.L., Green, C.S., and Bavelier, D. Video games as a tool to train visual skills. Restor Neurol Neurosci 26, 4-5 (2008), 435-446.

2. Allen, L.E., Slater, M.E., Proffitt, R.V., Quarton, E. \& Pelah, A.A. A new perimeter using the preferential looking response to assess peripheral visual fields in young and developmentally delayed children. J. Am. Assoc. Pediatric Ophthalmology and Strabismus 16, 3(2012), 261-265.

3. Bartle, D., Rossoff, S., Whittaker, D., Gooch, B., Kerns, K., and MacSween, J. Cognitive Games as Therapy for Children with FAS. In: Proc. of SIGGRAPH 2010, ACM (2010).

4. Castel, A.D.,Pratt, J., and Drummond, E. The effects of action video game experience on the time course of inhibition of return and the efficiency of visual search. Acta Psychologica 119, (2005), 217-230.

5. Ching, N., Wang, M., Ryan, M., Stevenson, R., and Andreallo, C. Beachcomber: A game for the visually impaired. In: Proc. of FDG 2011, (2011), 289-291. 
6. Coyle, D., McGlade, N., Doherty, G., and O'Reilly, G. Exploratory Evaluations of a Computer Game Supporting Cognitive Behavioural Therapy for Adolescents. In: Proc. of CHI 2011, (2011), 2937-2946.

7. Csíkszentmihályi, M. (1990). Flow: The Psychology of Optimal Experience. New York: Harper and Row.

8. Dutton, G.N., McKillop, E.C.A., and Saidkasimova, S. Visual problems as a result of brain damage in children. Br J Ophthalmol 90, 8 (2006), 932-933.

9. Eriksson, Y., and Gärdenfors, D. Computer games for children with visual impairments. In: Proc. of ICDVRAT 2004, (2004), 79-86.

10. Flores, E., Tobon, G., Cavallaro, E., Cavallaro, F. I., Perry, J. C., \& Keller, T. Improving patient motivation in game development for motor deficit rehabilitation. In Proc ACE 2008, ACM Press (2008) 381-384.

11. Gotsis, M., Piggot, J., Hughes, D., and Stone, W. SMART-Games: A Video Game Intervention for Children with Autism Spectrum Disorders. In: Proc. of IDC 2010, ACM (2010), 194-197.

12. Griffiths, M. (2003). The therapeutic use of videogames in childhood and adolescence. Clinical Child Psychology and Psychiatry, 8, 547-554.

13. Habgood, M. J., \& Ainsworth, S. E. (2011). Motivating children to learn effectively: Exploring the value of intrinsic integration in educational games. Journal of the Learning Sciences, 20, 169-206.

14.Han, L., Law-Gibson, D., and Reding, M. Key neurological impairments influence function-related group outcomes after stroke. Stroke 33, 7 (2002), 19201924.

15. Henderson, V., Lee, S., Brashear, H., Hamilton, H., Starner, T., and Hamilton, S. Development of an American Sign Language Game for Deaf Children. In: Proc. of IDC 2005, ACM (2005), 70-79.

16. Hernandez, H. A., Ye, Z., Graham, T. C., Fehlings, D., $\&$ Switzer, L. Designing action-based exergames for children with cerebral palsy. In Proc CHI 2013, ACM Press (2013), 1261-1270.

17. Kasten, E., Poggel, D.A., and Sabel, B.A. Computerbased training of stimulus detection improves color and simple pattern recognition in the defective field of hemianopic subjects. J Cogn Neurosci 12, 6 (2000), 1001-1012.

18. Kato, P.M., Cole, S.W., Bradlyn, A.S., and Pollock, B.H. A Video Game Improves Behavioral Outcomes in Adolescents and Young Adults With Cancer: A Randomized Trial. Pediatrics 122, 2 (2008), 305-317.

19. Kerkhoff, G., Münssinger, U., and Meier, E.K. Neurovisual rehabilitation in cerebral blindness. Arch Neurol 51, 5 (1994), 474-481.
20.Leat, S.J., Yadav, N.K., and Irving, E.L. Development of visual acuity and contrast sensitivity in children. $J$ Optom 2, 1 (2009), 19-26.

21.Li, R.W., Ngo, C., Nguyen, J., and Levi, D.M. VideoGame Play Induces Plasticity in the Visual System of Adults with Amblyopia. PLoS Biology 9, 8 (2011).

22. Mannan, S.K., Pambakian, A.L., and Kennard, C. Compensatory strategies following visual search training in patients with homonymous hemianopia: an eye movement study. J Neurol 257, 11 (2010), 18121821.

23. Morelli, T., Foley, J., Columna, L., Lieberman, L., and Folmer, E. VI-Tennis: a Vibrotactile/Audio Exergame for Players who are Visually Impaired. In: Proc. of FDG 2010, ACM (2010), 147-154.

24. Morelli, T., Foley, J., and Folmer, E. VI-Bowling: A Tactile Spatial Exergame for Individuals with Visual Impairments. In: Proc. of ASSETS'10, ACM (2010), 179-186.

25.Pambakian, A.L.M., Mannan, S.K., Hodgson, T.L., Kennard, C. Saccadic visual search training: a treatment for patients with homonymous hemianopia. $J$ Neurol Neurosurg Psychiatry 75, 10 (2004), 1443-1448.

26. Peli, E. Field expansion for homonymous hemianopia by optically induced peripheral exotropia. Optom Vis Sci 77, 9 (2000), 453-464.

27. Rector, K., Bennet, C.L., and Kientz, J.A. Eyes-Free Yoga: An Exergame Using Depth Cameras for Blind \& Low Vision Exercise. In: Proc. of ASSETS'13, ACM (2013).

28. Reinhard, J., Schreiber, A., Schiefer, U., Kasten, E., Sabel, B.A., Kenkel, S., Vonthein, R., and TrauzettelKlossinski, S. Does visual restitution training change absolute homonymous visual field defects? A fundus controlled study. Br J Ophthalmol 89, 1 (2005), 30-35.

29. Salen, K. and Zimmerman, E. Rules of Play: Game Design Fundamentals. MIT Press, Cambridge, 2004.

30. Schuett, S. The rehabilitation of hemianopic dyslexia. Nat Rev Neurol 5, 8 (2009), 427-437.

31. Smeddinck, J., Siegel, S., and Herrlich, M. Adaptive Difficulty in Exergames for Parkinson's disease Patients. In: Proc. of GI 2013, ACM (2013).

32. Sweetser, P., and Wyeth, P. GameFlow: A Model for Evaluating Player Enjoyment in Games. Computers in Entertainment 3, 3 (2005), Article 3A.

33. Treisman, A.M., and Gelade, G. A feature-integration theory of attention. Cognitive Psychol 12, 1 (1980), 97136.

34. Warren, M. Pilot study on activities of daily living limitations in adults with hemianopia. Am J Occup Ther 63, 5 (2009) 626-633. 